\title{
Biometric verification based on grip-pattern recognition
}

\author{
Raymond Veldhuis, Asker Bazen, Joost Kauffman, Pieter Hartel \\ University of Twente, Faculty of Electrical Engineering, Mathematics and Computer Science \\ P.O. box 217, 7500 AE Enschede, The Netherlands
}

\begin{abstract}
This paper describes the design, implementation and evaluation of a user-verification system for a smart gun, which is based on grip-pattern recognition. An existing pressure sensor consisting of an array of $44 \times 44$ piezoresistive elements is used to measure the grip pattern. An interface has been developed to acquire pressure images from the sensor. The values of the pixels in the pressure-pattern images are used as inputs for a verification algorithm, which is currently implemented in software on a PC. The verification algorithm is based on a likelihoodratio classifier for Gaussian probability densities. First results indicate that it is feasible to use grip-pattern recognition for biometric verification.
\end{abstract}

Keywords: Biometric verification, likelihood ratio, smart gun, grip-pattern recognition.

\section{INTRODUCTION}

Nowadays there is a growing interest in personalized applications that use biometrics as an access key. Wellknown methods use fingerprints, hand geometry, iris scans, or voice characteristics to identify a person or to verify a person's identity. Since technology is improving and becoming more affordable, biometrics is becoming more popular for daily use. Powerful processors provide the possibility of doing complex calculations on large sets of data within a short time. This creates new possibilities for high-speed verification or even identification for many everyday applications.

This paper describes part of the development of a security system for a personalized handgun, a so-called 'smart gun', that makes use use of biometric verification. The biometric features that are used are those of the twodimensional pattern of the pressure that is exerted on the gun's butt. This pressure pattern will be further referred to as the grip pattern. The goal of the ongoing research is to contribute to a weapon that can only be fired by the rightful user.

The smart-gun concept receives great interest in the US, where weapon safety is an important issue. The technology described here might help to prevent many accidents at home, where young children get to play with their parent's guns. ${ }^{1,2}$ Also the police (in the US as well as elsewhere) show interest, since carrying a gun in public brings considerable risks. In the US vital statistics show that about $8 \%$ of the law-enforcement officers, who are killed in a shooting incident, are shot with their own weapon. ${ }^{3}$

Today there are already several types of smart guns available on the market varying from simple trigger locks ${ }^{4}$ to more advanced electronically or magnetically controlled systems. ${ }^{5,6}$ These systems are in general not personalized, because they are controlled by a transmissible magnetic or electronic key. They may also be vulnerable to interference and illegal access with forged keys.

This paper describes the first steps of the development and the testing of a prototype biometric security system for a smart gun based on grip-pattern recognition. Section 2 briefly describes the system's hardware. The algorithm for the biometric verification is discussed in Section 3. Subsequently, a first experimental evaluation the system and its results, which are promising, are explained in Section 4. Finally, the conclusions are presented in Section 5.

Further author information: (Send correspondence to Raymond Veldhuis)

E-mail: R.N.J.Veldhuis@utwente.nl, Telephone: +31(0)534892838 


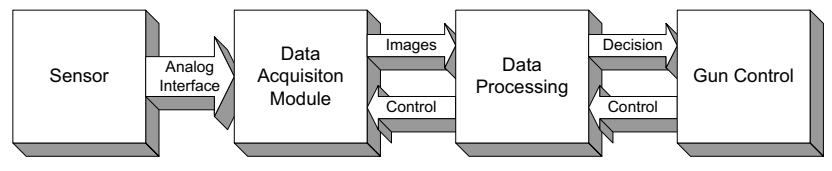

Figure 1. High-level description of the smart-gun verification system.

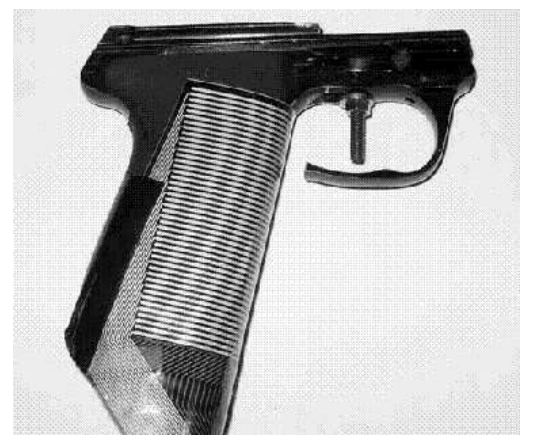

Figure 2. The sensor wrapped around the gun's butt.

\section{HARDWARE}

The smart-gun verification system can be subdivided into the following 4 blocks: Sensor, Data-Acquisition Module (DAM), Data Processing (DP), and Gun Control (GC). These blocks are shown in Fig. 1 and briefly discussed below.

The DAM consists of an analog measuring circuit, which is controlled by digital logic. The acquired data is currently transmitted to a PC (DP), which processes the data with a verification algorithm. The Gun Control, responsible for controlling the locking mechanism, has not been implemented yet. The depicted control signals can be used to activate and manage the electronics for power saving purposes.

The sensor that is used for this project is a piezo-resistive pressure sensor made by the company Tekscan Inc ${ }^{7}$ with a range of $0-30$ PSI. It is available in a size that fits the prototype gun butt, which is that of a Walther P5, see Fig. 2. This sensor consists of two layers of strong and flexible polyester foil. Each layer has 44 silver electrode strips deposited on one side. One layer has vertical and the other horizontal strips. A piezo-resistive ink has been printed on top of the silver leads. This construction results in a network of silver strips with a resistive element at each crossing. The entire sensor array can be modelled as a $44 \times 44$ network of variable resistors. Fig. 3 shows a schematic diagram of the sensor construction (left) and an example of the resistor-network model containing $4 \times 4$ resistors (right). The resistive elements are sensitive to pressure and have a resistance of more than $5 \mathrm{M} \Omega$ at zero load and about $20 \mathrm{k} \Omega$ at full load.

The grip pattern is measured by determining each resistor value. This is done by subsequently connecting the horizontal and vertical conductors to an analog measuring circuit. The connections can be altered by multiplexers controlled by digital logic. The analogue and digital circuitry are described in detail in Ref. 8. After analog-todigital conversion, the pixels in the pressure pattern are represented by 8-bit integers.

\section{VERIFICATION ALGORITHM}

The verification algorithm is based on a likelihood-ratio classifier for Gaussian probability densities. ${ }^{9,10}$ The values of the pixels of the pressure patterns are the features for a verification algorithm. Figure 4 shows the average grip patterns of 4 users. The grip patterns shown are clearly different.

The pixel values are arranged in a (in this case $44 \times 44=1936$-dimensional) column vector $\mathbf{x}$. The feature vector $\mathbf{x}$ is normalized, i.e. $\|\mathbf{x}\|_{2}=1$, prior to classification. The reason for this normalization is twofold. First, it 

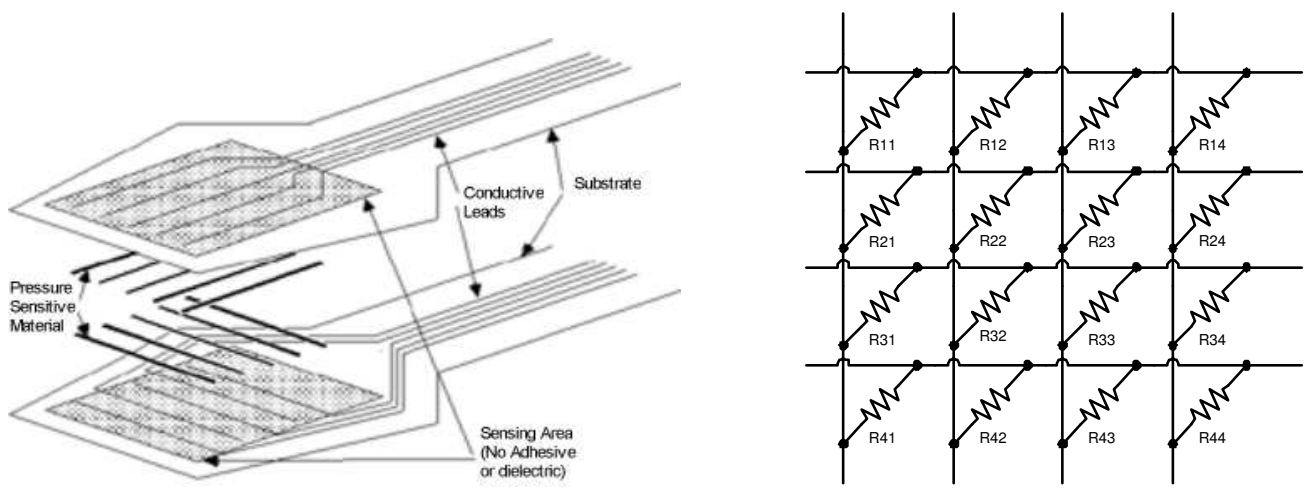

Figure 3. Schematic diagram of the sensor construction ${ }^{7}$ (left) and an example of the resistor-network model containing $4 \times 4$ resistors (right).
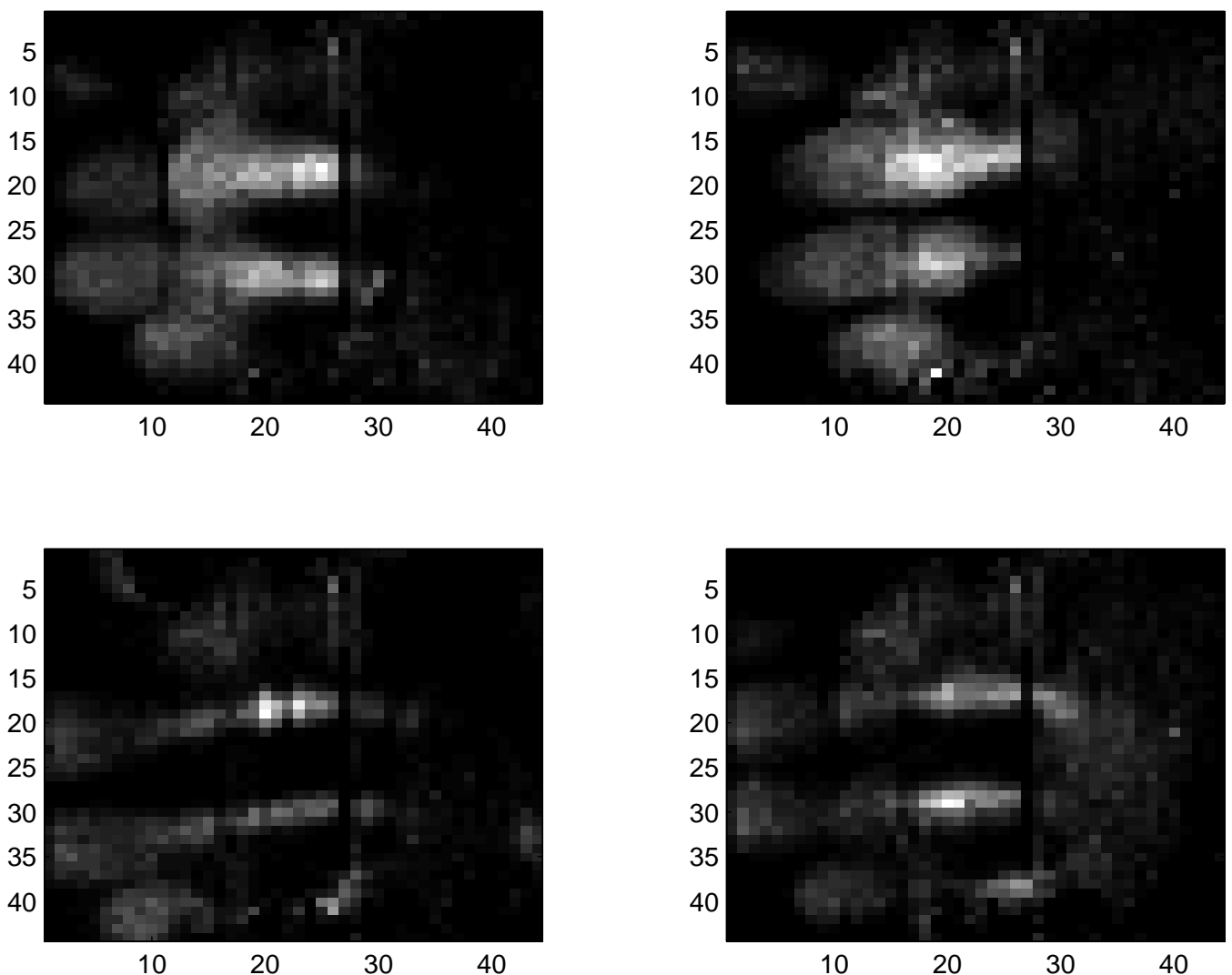

Figure 4. Gray-scale images of the average grip patterns of 4 users. 
compensates for the aging of the sensor, due to which the resistance changes with time. Second, it reduces the variations of the grip patterns, which is beneficial for the recognition results.

Measured data can originate form a genuine user or from an impostor. The genuine data of a specific user is characterized by a mean $\mu_{\mathrm{W}}$ and a covariance matrix $\boldsymbol{\Sigma}_{\mathrm{W}}$, where the subscript W denotes 'Within-class', while the impostor data is characterized by $\mu_{\mathrm{T}}$ and $\boldsymbol{\Sigma}_{\mathrm{T}}$, where the subscript T denotes 'Total'. The similarity score $S(\mathbf{x})$ to the genuine user of a measurement $\mathbf{x}$ is derived from the log-likelihood ratio. It is calculated by

$$
S(\mathbf{x})=-\left(\mathbf{x}-\mu_{\mathrm{W}}\right)^{\prime} \boldsymbol{\Sigma}_{\mathrm{W}}^{-1}\left(\mathbf{x}-\mu_{\mathrm{W}}\right)+\left(\mathbf{x}-\mu_{\mathrm{T}}\right)^{\prime} \boldsymbol{\Sigma}_{\mathrm{T}}^{-1}\left(\mathbf{x}-\mu_{\mathrm{T}}\right) .
$$

The ' denotes matrix or vector transposition. If $S(\mathbf{x})$ is above a predetermined threshold $T$, the user is accepted, otherwise he is rejected.

In practice, the means and covariance matrices are unknown and have to be estimated from a set of training data. In our case, the sensor network has $44 \times 44=1936$ nodes, and the feature vectors have equally many elements. Therefore, the number of examples needs to be greater than 1936 in order to prevent the covariance matrices from being singular and much greater than 1936 for a good estimate. Evidently, this large number of measurements would be impractical for user enrollment. Moreover, even if enough measurements would be available, the evaluation of (1) would, with 1936-dimensional feature vectors, still be too high a computational burden.

These problems are solved by whitening the feature space and at the same time reducing its dimensions. The first step is a Principal Component Analysis (PCA), ${ }^{11}$ determining the most important dimensions (with the greatest variance) by doing a singular value decomposition (SVD) on the matrix $\mathbf{X}$, of which the columns are the feature vectors in the training set. The data matrix $\mathbf{X}$ has $N_{\text {raw }}=1936$ rows and $N_{\text {ex }}$ columns. The columns are training examples taken from $N_{\text {user }}$ users. We assume that $\mathbf{X}$ has zero column mean. If necessary, the column mean has to be subtracted from the data matrix prior to SVD. As a result of the SVD the data matrix $\mathbf{X}$ is written as

$$
\mathbf{X}=\mathbf{U}_{\mathbf{X}} \mathbf{S}_{\mathbf{X}} \mathbf{V}_{\mathbf{X}}^{\prime}
$$

with $\mathbf{U}_{\mathbf{X}}$ an $N_{\text {raw }} \times N_{\text {ex }}$ orthonormal matrix spanning the column space of $\mathbf{X}, \mathbf{S}_{\mathbf{X}}$ an $N_{\text {ex }} \times N_{\text {ex }}$ diagonal matrix of which the (non-negative) diagonal elements are the singular values of $\mathbf{X}$ in descending order, and $\mathbf{V}_{\mathbf{X}}$ an $N_{\text {ex }} \times N_{\text {ex }}$ orthonormal matrix spanning the row space of $\mathbf{X}$. The whitening and the first dimension-reduction step are achieved as follows. Let the $N_{\text {raw }} \times N_{\mathrm{PCA}}$ matrix $\mathbf{U}_{\mathrm{PCA}}$ be the submatrix of $\mathbf{U}$ consisting of the first $N_{\mathrm{PCA}}<N_{\mathrm{ex}}$ columns. Furthermore, let the $N_{\mathrm{PCA}} \times N_{\mathrm{PCA}}$ matrix $\mathbf{S}_{\mathrm{PCA}}$ be the first principal $N_{\mathrm{PCA}} \times N_{\mathrm{PCA}}$ submatrix of $\mathbf{S}$. Finally, let the $N_{\mathrm{ex}} \times N_{\mathrm{PCA}}$ matrix $\mathbf{V}_{\mathrm{PCA}}$ be the submatrix of $\mathbf{V}$ consisting of the first $N_{\mathrm{PCA}}$ columns. The whitened data matrix with reduced dimensions is now given by

$$
\mathbf{Y}=\sqrt{N_{\mathrm{ex}}-1} \mathbf{V}_{\mathrm{PCA}}^{\prime}
$$

The resulting dimension $N_{\mathrm{PCA}}$ must be chosen such that only the relevant dimensions, i.e. with sufficiently high corresponding singular values are kept. A minimum requirement is that all diagonal element of $\mathbf{S}_{\mathrm{PCA}}$ are strictly positive. The corresponding whitening transform is

$$
\mathbf{T}_{\text {white }}=\sqrt{N_{\mathrm{ex}}-1} \mathbf{S}_{\mathrm{PCA}}^{-1} \mathbf{U}_{\mathrm{PCA}}^{\prime} .
$$

Estimating the total mean and the total covariance matrix from $\mathbf{Y}$ would result into $\mu_{\mathrm{T}}=0$ and $\boldsymbol{\Sigma}_{\mathrm{T}}=$ $\frac{1}{N_{\mathrm{ex}}-1} \mathbf{Y} \mathbf{Y}^{\prime}=\mathbf{I}$.

The whitened matrix $\mathbf{Y}$ can now be used to estimate the within-class covariance matrices. Here we make the simplifying assumption that the within-class variations of all users are characterized by one within-class covariance matrix $\boldsymbol{\Sigma}_{\mathrm{W}}$. The reason is that often not enough user data are available to reliably estimate individual withinclass covariance matrices. First, note that the users' contributions to the training data can be ordered such that

$$
\mathbf{Y}=\left(\mathbf{Y}_{1}, \ldots, \mathbf{Y}_{N_{\text {user }}}\right),
$$

with $\mathbf{Y}_{i}$ the whitened data from user $i$. The column mean $\mu_{i}$ from $\mathbf{Y}_{i}$ estimates the mean feature vector of user $i$ after whitening. The matrix

$$
\mathbf{W}=\left(\mathbf{Y}_{1}-\mu_{1}, \ldots, \mathbf{Y}_{N_{\text {user }}}-\mu_{N_{\text {user }}}\right)
$$


contains all variations around the means. Note that the within-class covariance matrix after whitening can now be estimated by $\boldsymbol{\Sigma}_{\mathrm{W}}=\frac{1}{N_{\mathrm{ex}}-1} \mathbf{W} \mathbf{W}^{\prime}$, but instead we will proceed immediately to estimating a diagonalized version of $\boldsymbol{\Sigma}_{\mathrm{W}}$. A second SVD on $\mathbf{W}$ results in

$$
\mathbf{W}=\mathbf{U}_{\mathbf{W}} \mathbf{S}_{\mathbf{W}} \mathbf{V}_{\mathbf{W}}^{\prime}
$$

with $\mathbf{U}_{\mathbf{W}}$ an $N_{\mathrm{PCA}} \times N_{\mathrm{PCA}}$ orthonormal matrix spanning the column space of $\mathbf{W}, \mathbf{S}_{\mathbf{W}}$ an $N_{\mathrm{PCA}} \times N_{\mathrm{PCA}}$ diagonal matrix of which the (non-negative) diagonal elements are the singular values of $\mathbf{W}$ in descending order, and $\mathbf{V}_{\mathbf{W}}$ an $N_{\text {ex }} \times N_{\text {PCA }}$ orthonormal matrix spanning the row space of $\mathbf{W}$. The within-class covariance matrix can be diagonalized by pre-multiplying $\mathbf{W}$ by $\mathbf{U}_{\mathbf{W}}^{\prime}$. This transformation can also be applied to the whitened data $\mathbf{Y}$, and thus to the within-class means $\mu_{i}$, because it does not affect the whitening nor that $\boldsymbol{\Sigma}_{\mathrm{T}}=\mathbf{I}$. For the resulting, diagonal, within-class covariance matrix, further denoted by $\boldsymbol{\Lambda}$, we have

$$
\boldsymbol{\Lambda}=\frac{1}{N_{\mathrm{ex}}-1} \mathbf{S}_{\mathbf{W}}^{2}
$$

For the resulting within-class means, further denoted by $\nu_{i}$, we have

$$
\nu_{i}=\mathbf{U}_{\mathbf{W}}^{\prime} \mu_{i}, i=1, \ldots, N_{\mathrm{user}} .
$$

It can be shown, but this falls outside the scope of this paper, that

$$
\begin{aligned}
\boldsymbol{\Lambda}_{j, j} & =1, j=1, \ldots, N_{\mathrm{PCA}}-N_{\text {user }}+1 \\
\left(\nu_{i}\right)_{j} & =0, j=1, \ldots, N_{\mathrm{PCA}}-N_{\text {user }}+1 .
\end{aligned}
$$

This means that only the last $N_{\text {user }}-1$ dimensions of $\mathbf{U}_{\mathbf{W}}^{\prime} \mathbf{W}$ can contribute to the verification. Therefore, a further dimension reduction is obtained by discarding the first $N_{\mathrm{PCA}}-N_{\text {user }}+1$ dimensions in $\mathbf{U}_{\mathbf{W}}^{\prime} \mathbf{W}$. This can be achieved by pre-multiplying $\mathbf{W}$ by a transformation matrix $\mathbf{U}_{\mathrm{LDA}}^{\prime}$, with $\mathbf{U}_{\mathrm{LDA}}$ the submatrix of $\mathbf{U}_{\mathbf{W}}$ consisting of the last $N_{\text {user }}-1$ columns. The subscript LDA, which stands for Linear Discriminant Analysis, is used, because this operation is, in fact, a dimension reduction by means of LDA. ${ }^{11}$ The notations $\boldsymbol{\Lambda}$ and $\nu_{i}$ will be maintained for the within-class covariance matrix and the within-class means.

The sequence of transformations described above can be replaced by one multiplication by an $\left(N_{\text {user }}-1\right) \times N_{\text {raw }}$ matrix, denoted by

$$
\mathbf{T}=\sqrt{N_{\mathrm{ex}}-1} \mathbf{U}_{\mathrm{LDA}}^{\prime} \mathbf{S}_{\mathrm{PCA}}^{-1} \mathbf{U}_{\mathrm{PCA}}^{\prime} .
$$

Let $\mathbf{y}=\mathbf{T} \mathbf{x}$ denote a transformed input feature vector, then the similarity score (1) computed for user $i$ becomes

$$
S_{i}(\mathbf{y})=-\left(\mathbf{y}-\nu_{i}\right)^{\prime} \boldsymbol{\Lambda}^{-1}\left(\mathbf{y}-\nu_{i}\right)+\mathbf{y}^{\prime} \mathbf{y} .
$$

Because $\boldsymbol{\Lambda}$ is a diagonal matrix and has much smaller dimensions $\left(\left(N_{\text {user }}-1\right) \times\left(N_{\text {user }}-1\right)\right)$ than the original covariances matrices $\left(N_{\text {raw }} \times N_{\text {raw }}\right)$ in (1), the number of computations has decreased considerably. In the experiments that are described in the following section, we have that $N_{\text {user }}=26$ and $N_{\text {raw }}=1936$. This means that the number of operations needed to evaluate (13) in combination with the transform (12) is about a factor of 800 less than the number of operations needed to evaluate (1). Nearly all of the computational burden is in the computation of the transform (12).

A further dimension reduction is possible, either by a user-independent transform by means of LDA, or by a user-dependent transform, such as the new Maximum Divergence Analysis (MDA) technique, which has been developed by the authors. Both techniques will reduce the computational load and may have a positive effect on the verification performance on a test set which is different from the training set, but will not be discussed in this contribution. 


\section{EXPERIMENTAL RESULTS}

As described in Section 3 a decision is made by comparing the similarity score (13) to a threshold $T$. If $S(\mathbf{y})>T$, then the user is accepted, otherwise he is rejected. This may lead to two types of errors: the false acceptance of an impostor and the false rejection of an authorized user. The probability of a false acceptance is the FalseAcceptance Rate (FAR). The probability of a false rejection is the False-Reject Rate (FRR). There is a trade-off between FAR and FRR, governed by the choice of $T$. The Equal-Error Rate (EER), i.e. the value of FAR and FRR when $T$ is such that FAR and FRR are equal, is often used as a performance measure.

Before the performance can be assessed, the parameter $N_{\mathrm{PCA}}$ has to be set. Although the feature vector is always reduced to a dimension $N_{\text {user }}-1$, the verification performance depends on $N_{\text {PCA }}$. The optimal value has been found experimentally by determining the system's (EER) for different values of $N_{\mathrm{PCA}}$. As a (flat) optimum we found $N_{\mathrm{PCA}}=3 N_{\mathrm{user}}$.

\subsection{Method}

For the experiment a collection of 855 handgrip patterns was gathered from a group of 26 mostly untrained subjects. From each subject 30 to 100 right-hand grip images were taken. Between every three measurements the subject was asked to completely renew his grip by releasing the gun and retaking it. The three measurements for the same grip register variations in the pressure while holding the same grip, since it is quite impossible to maintain a constant (within the DAM's precision) grip-pressure distribution. The renewal of the grip is necessary to register variations in the grip.

The data were randomly split into a training and a test set of, nearly, equal sizes. This was done in such a way that the three measurements for the same grip were kept together.

The total mean, the diagonal within-class covariance matrix $\boldsymbol{\Lambda}(8)$, the within-class means $\nu_{i}(9)$ and the total transform matrix $\mathbf{T}$ (12) were estimated from the training set. Similarity scores (13) were computed for all the data in the training and in the test set. For each user the similarity scores were divided into genuine matching scores, i.e. the $\mathbf{y}$ and the $\nu_{i}$ in (13) correspond to the same user, and impostor matching scores, i.e. the $\mathbf{y}$ and the $\nu_{i}$ in (13) correspond to different users. This resulted in 15-50 genuine matching scores and 805-840 impostor matching scores per user.

\subsection{Results and discussion}

Both the training and test sets were used to determine the EER for each user, the average EER and the overall EER. The latter is the EER that is obtained when all the genuine similarity scores are taken together and all the impostor similarity scores are taken together. This would be the EER if the same threshold $T$ were used for all users. The results are shown in Table 1.

The verification results obtained on the training set seem perfect. This seems also the case for the results for 16 users obtained on the test set. An average EER of $1.8 \%$ is reasonable, but probably not yet good enough for the application in a police gun, where the FRR should be extremely low. In The Netherlands the probability of malfunctioning of a police gun must be below $10^{-4}$. Nevertheless, these results are promising and they clearly indicate that the grip pattern contains sufficient information that can be used for verification. However, it is also true that not enough data were available to reliably estimate the lower EERs and that the current values for the EERs are not precise enough to make a well-founded statement about the performance of the system. To achieve this, more data are needed from a greater population and with more scans per subject. Probably another important aspect, that determines the outcome's precision, is the (lack of) shooting experience of the subjects. It appeared that the more experienced subjects (who had handled the gun more often and over a longer period) always had perfect verification results. To approximate realistic situations, the data should be collected from experienced subjects at a shooting range. 
Table 1. The EER for each user, the average EER and the overall EER.

\begin{tabular}{|r|r|r|}
\hline User & Training & Test \\
\hline 1 & 0 & 0 \\
2 & 0 & 0.0406 \\
3 & 0 & 0 \\
4 & 0 & 0 \\
5 & 0 & 0 \\
6 & 0 & 0.0418 \\
7 & 0 & 0.0382 \\
8 & 0 & 0 \\
9 & 0 & 0 \\
10 & 0 & 0.0588 \\
11 & 0 & 0 \\
12 & 0 & 0.0406 \\
13 & 0 & 0 \\
14 & 0 & 0.0673 \\
15 & 0 & 0.0358 \\
16 & 0 & 0.0564 \\
17 & 0 & 0.0661 \\
18 & 0 & 0 \\
19 & 0 & 0 \\
20 & 0 & 0 \\
21 & 0 & 0 \\
22 & 0 & 0.0576 \\
23 & 0 & 0 \\
24 & 0 & 0 \\
25 & 0 & 0 \\
26 & 0 & 0 \\
\hline Average & 0 & 0.0177 \\
Overall & 0 & 0.0515 \\
\hline
\end{tabular}

\section{CONCLUSIONS}

The current hardware implementation has proven to be useful for the first experiments and demonstrations. The piezo-resistive sensor array of the Tekscan sensor has been found suitable for detecting handgrip squeeze patterns and appeared to be a good option for a low-cost experimental setup. The current system uses a PC for the implementation of the verification algorithm. Though the training of the system requires some extensive computations, the verification part is quite straightforward and suitable for an efficient hardware implementation.

The test results indicate that the grip pattern contains sufficient information that can be used for verification. The current values for the EERs are not precise enough to make a well-founded statement about the performance of the system. This is caused by the limited number of data that was collected for training and testing.

Further improvements can be expected from a better modelling of the within-class covariance matrix of the intended user group, i.e. the police. This is based on the observation that experienced marksmen show very little variation in the way the hold their weapon. It is also worth investigating a further dimension reduction to a value below the number of users. This is likely to improve the performance on the test set and thus in the real world.

\section{REFERENCES}

1. D. L. Hoyert., E. Arias, B. L. Smith, S. L. Murphy, and K. D. Kochanek, "National Vital Statistics Reports," Volume 49 Number 8, NVSS, September 212001.

2. Anonymous, "Factsheet: Firearm Injury and Death in the United States," tech. rep., Johns Hopkins University; Center for Gun Policy and Research, 2000. 
3. The national Uniform Crime Reporting Program, "Law Enforcement Officers Killed and Assaulted," tech. rep., Federal Bureau of Investigation, 2001.

4. C. D. Corporation, "Saf-t-block." Web Page, Januari 2003. http://members.aol.com/saftblok/.

5. S. L. T. Inc., "Magloc." Web Page, Januari 2003. http://www. smartlock.com/.

6. Peter Wetzig, "The World's First Totally Electronic Hand Gun," Metal Storm Limited, April 2000. http: //www.metalstorm.com/12_odwyervle/smartgun.html.

7. W. L. Maness et al., "Pressure and contact sensor for measuring dental occlusion." United States Patent; 4,856,993, Aug. 1989. Tekscan, Inc.

8. J. Kauffman, A. Bazen, S. Gerez, and R. Veldhuis, "Grip-pattern recognition for smart guns," in Proceedings of ProRISC 2003, 14th Annual Workshop on Circuits, Systems and Signal Processing, pp. 379-384.

9. H. V. Trees, Detection, estimation, and modulation theory, Wiley, New York, 1968.

10. A. Bazen and R. Veldhuis, "Likelihood-ratio-based biometric verification," To appear in: IEEE Transactions on Circuits and Systems for Video Technology 14, 2004.

11. Richard O. Duda and Peter E. Hart and David G. Stork, Pattern Classification (2nd Edition), WileyInterscience, 2000. 